\title{
MARKOV PROPERTIES OF BROWNIAN LOCAL TIME
}

\section{BY DAVID WILLIAMS ${ }^{1}$}

Communicated by Henry McKean, Jr., April 11, 1969

1. Starred references are to Itô and McKean [1], the terminology and notation of which are used here. Our aim is to indicate an elementary approach to local time theory which highlights certain Markov properties discovered by Ray and Knight (\$2.8*). Details and further applications of our method will be given elsewhere.

Let $X=\{x(t): t \geqq 0\}$ be standard Brownian motion and, for $t \geqq 0$, set

$$
\begin{aligned}
& \phi(t)=\operatorname{measure}\{s: 0 \leqq s \leqq t ; x(s) \geqq 0\}, \\
& \rho(t)=\inf \{s: \phi(s)>t\}, \quad y(t)=x(\rho(t)) .
\end{aligned}
$$

Then $Y=\{y(t): t \geqq 0\}$ is a reflecting Brownian motion $\left(\$ 2.11^{*}\right)$ with local time

$$
L(t)=\lim _{\epsilon \downarrow 0}(2 \epsilon)^{-1} \text { measure }\{s: 0 \leqq s \leqq t, 0 \leqq y(s)<\epsilon\} .
$$

ThEOREM 1. For $x \geqq 0, t \geqq 0$ and $\theta>0$,

$$
E_{x}\{\exp [-\theta \rho(t)] \mid Y\}=\exp \left[-\theta t-(2 \theta)^{1 / 2} L(t)\right] .
$$

Notes. Lévy's arc-sine law ( $\$ 2.6^{*}$, Equation (17)) follows quickly on taking expectations of both sides of Equation (1). Excursion theory $\left(\$ 2.9^{*}\right)$ renders Theorems 1 and 2 intuitively clear.

Proof. Introduce an exponential variable $\zeta$ (with rate $\theta$ ) independent of $X$. Set

$$
\phi^{*}(t)=\phi(t \wedge \zeta), \quad \rho^{*}(t)=\inf \left\{s: \phi^{*}(s)>t\right\}, \quad y^{*}(t)=x\left(\rho^{*}(t)\right) .
$$

Then $Y^{*}=\left\{y^{*}(t): t<\phi(\zeta)\right\}$ is a diffusion on $[0, \infty)$ with generator

$$
G^{*} f=\frac{1}{2} f^{\prime \prime}-\theta f \quad \text { on } \quad C^{2}[0, \infty) \cap\left\{f: f^{+}(0)=\gamma f(0)\right\},
$$

where $\gamma=(2 \theta)^{1 / 2}$. Thus the transition function $P^{*}$ of $Y^{*}$, which induces corresponding measures

$$
P_{x}^{*}(x \geqq 0) \quad \text { on } \quad B[Y]=B[y(s): s \geqq 0],
$$

is of the form

$$
P^{*}(t)=\exp (-\theta t) P \cdot(t)
$$

\footnotetext{
1 This work was done at the University of Cambridge.
} 
where $P \cdot$ is the transition function of elastic Brownian motion ( $\left.\$ 2.3^{*}\right)$ with killing constant $\gamma$. The consequent identity

$$
E_{x}\{\exp [-\theta \rho(t)] ; B\}=P_{x}^{*}\{B\}=E_{x}\{\exp [-\theta t-\gamma L(t)] ; B\},
$$

where $x \geqq 0$ and $B \in B[Y]$, implies Theorem 1 .

2. Let $T$ be standard Brownian local time and fix $b=-c<0$. Let $Z$ be the process on $\{b\} \cup[0, \infty)$ obtained from $X$ by using $T(t, b)$ $+\phi(t)$ as new time parameter. The generator $A$ of $Z$ has domain

$$
D(A)=\left\{f: f \in C^{2} \quad \text { on }[0, \infty) ; f(0)-f(b)=c f^{+}(0)\right\}
$$

and, for $f$ in $D(A)$,

$$
(A f)(b)=[f(0)-f(b)] / c, \quad(A f)(x)=\frac{1}{2} f^{\prime \prime}(x) \text { on }(0, \infty) .
$$

Repeat the argument of $\S 1$ with $Z$ replacing $X$. One finds that the new $Y^{*}$ has killing constant $\gamma=\theta /(1+c \theta)$ and thus obtains

TheOREM 2. For positive constants $x, \tau$ and $\theta$,

$$
E_{x}\{\exp [-\theta T(\rho(\tau), b)] \mid Y\}=\exp [-\theta L(\tau) /(1+c \theta)] .
$$

Theorem 3 is a measure-theoretic consequence.

Theorem 3. Equation (2) remains valid if $\tau$ is a positive random variable measurable with respect to $B[Y]$.

Ray's theorem that

$$
\left\{2 T\left(\tau_{1}, 1-b\right): 0 \leqq b \leqq 1, P_{0}\right\} \quad\left(\tau_{1}=\inf \{s: x(s)=1\}\right)
$$

is the square of a 2-dimensional Bessel process follows easily from Theorem 3.

\section{REFERENCE}

1. K. Ito and H. P. McKean, Jr., Diffusion processes and their sample paths, Die Grundlehren der Mathematischen Wissenschaften, Band 125, Academic Press, New York and Springer-Verlag, Berlin, 1965.

University College, Swansea, Great Britain 